\title{
Recombination hotspots and host susceptibility modulate the adaptive value of recombination during maize streak virus evolution
}

\author{
Adérito L Monjane ${ }^{1}$, Eric van der Walt ${ }^{2}$, Arvind Varsani ${ }^{3,4,5}$, Edward P Rybicki ${ }^{1,6}$ and Darren P Martin ${ }^{6,7^{*}}$
}

\begin{abstract}
Background: Maize streak virus -strain A (MSV-A; Genus Mastrevirus, Family Geminiviridae), the maize-adapted strain of MSV that causes maize streak disease throughout sub-Saharan Africa, probably arose between 100 and 200 years ago via homologous recombination between two MSV strains adapted to wild grasses. MSV recombination experiments and analyses of natural MSV recombination patterns have revealed that this recombination event entailed the exchange of the movement protein - coat protein gene cassette, bounded by the two genomic regions most prone to recombination in mastrevirus genomes; the first surrounding the virion-strand origin of replication, and the second around the interface between the coat protein gene and the short intergenic region. Therefore, aside from the likely adaptive advantages presented by a modular exchange of this cassette, these specific breakpoints may have been largely predetermined by the underlying mechanisms of mastrevirus recombination. To investigate this hypothesis, we constructed artificial, low-fitness, reciprocal chimaeric MSV genomes using alternating genomic segments from two MSV strains; a grass-adapted MSV-B, and a maize-adapted MSV-A. Between them, each pair of reciprocal chimaeric genomes represented all of the genetic material required to reconstruct - via recombination - the highly maize-adapted MSV-A genotype, MSV-MatA. We then coinfected a selection of differentially MSV-resistant maize genotypes with pairs of reciprocal chimaeras to determine the efficiency with which recombination would give rise to high-fitness progeny genomes resembling MSV-MatA.
\end{abstract}

Results: Recombinants resembling MSV-MatA invariably arose in all of our experiments. However, the accuracy and efficiency with which the MSV-MatA genotype was recovered across all replicates of each experiment depended on the MSV susceptibility of the maize genotypes used and the precise positions - in relation to known recombination hotspots - of the breakpoints required to re-create MSV-MatA. Although the MSV-sensitive maize genotype gave rise to the greatest variety of recombinants, the measured fitness of each of these recombinants correlated with their similarity to MSV-MatA.

Conclusions: The mechanistic predispositions of different MSV genomic regions to recombination can strongly influence the accessibility of high-fitness MSV recombinants. The frequency with which the fittest recombinant MSV genomes arise also correlates directly with the escalating selection pressures imposed by increasingly MSV-resistant maize hosts.

\section{Background}

Viruses are characteristically capable of rapid evolutionary adaptation. Typically, the primary driver of this adaptation is high basal mutation rates. For example, single-stranded RNA and DNA virus genomes generally

\footnotetext{
* Correspondence: darrin.martin@uct.ac.za

${ }^{6}$ Computational Biology Group, Institute of Infectious Disease and Molecular Medicine, University of Cape Town, Observatory, 7925, Cape Town, South Africa

Full list of author information is available at the end of the article
}

accumulate $10^{-4}$ to $10^{-6}$ mutations per site per replication cycle [1-5]. Many viruses also experience high rates of homologous recombination and/or reassortment of genome components [6-10]. Acting either individually or in concert, these processes can create novel combinations of new and pre-existing genetic polymorphisms, generating substantial genetic diversity within a single, closely-related group of viruses (such as those within the same species $[11,12]$ ), or among viruses belonging to more distantly-related species, genera, or even families.

\section{() Biomed Central}


In this way, new virus strains [13], species [14], genera $[15,16]$, or - in at least some instances - families $[17,18]$ can be formed. It is probably this capacity for rapid genetic diversification that has enabled the recent emergence of numerous economically and socially important pathogenic viruses of humans and their domesticated plants and animals.

One of these emergent pathogens is Maize streak virus strain A (MSV-A), which apparently arose around the mid 1800 s via a recombination event between two Digitaria $s p$. adapted MSVs $[3,13]$. MSV-A is distributed throughout sub-Saharan Africa where it jeopardizes sustainable maize production in some of the world's poorest countries [19-21]. Its single component, circular, $\sim 2.7 \mathrm{~Kb}$, single-stranded DNA (ssDNA) genome encodes a movement protein (MP) and coat protein (CP) in the virion-sense [22-24], and in the complementary-sense the replication-associated proteins Rep and RepA [25-29]. Separating the virion- and complementary-sense open reading frames (ORFs) are the long intergenic region (LIR) - comprising transcriptional promoter elements and the virion strand origin of replication (v-ori) [30] - and a short intergenic region (SIR), where the transcription termination elements and the complementary strand origin of replication reside.

Experimental evolution can reveal key aspects of natural evolution, and has been used to study evolutionary processes leading to, for example, viral host-switching [31], resistance-breakage [32], and increased virulence [33]. With their small genome size, recombinogenic nature, and high mutation rates, geminiviruses have proved to be excellent models for experimental studies of the evolutionary mechanisms of virus emergence and adaptation. Accordingly, various experiments involving geminiviruses - and MSV in particular - have illuminated genetic factors underpinning important evolutionary processes, including the adaptation of these viruses to specific vector species [34] or hosts [35-37], their mutational dynamics [2,3,38-41], and the biochemical and selective factors constraining their adaptation through recombination [37,42-45].

We have previously described an experimental scheme for studying factors that affect the adaptive potential of recombination in mastrevirus evolution [45]. In this scheme, low-fitness laboratory-constructed reciprocal chimaeras of two wild-type (wt) MSV isolates (one naturally adapted to wild grasses, and the other adapted to maize) are co-introduced into a host plant, where they might re-create - via recombination - relatively high-fitness genomes that approximate the fittest wt genome. We used this experimental approach to identify maizeadaptive genetic polymorphisms within the MSV-A genome, and verified apparent hotspots of recombination detected in natural MSV populations at the v-ori and
SIR $[13,46]$, indicating that these regions of the genome are mechanistically predisposed to recombination. Moreover, this study demonstrated that a pair of coinfected low-fitness MSV genomes could efficiently recombine to regenerate genomes closely resembling wt MSV-A genotypes, and displaying fitness in maize that approached that of field-isolated MSV-A viruses.

In this previous study, only MSV-A-like genomes were recovered, suggesting that genomes representing positions of intermediate fitness within the sequence space were substantially less maize-adapted than the MSV-Alike genomes. This may have been due to the severe selective constraints of the MSV-resistant maize genotypes and/or to the specific pairs of chimaeric viruses used. Either way, these constraints limited the power and resolution of that specific experimental scheme. For example, the scheme provided no plausible way in which to recapitulate the evolutionary path that prototypical MSV-A genomes circulating in the mid 1800s may have taken - presumably via mutation and recombination, in a variety of differentially MSV-resistant maize plants - to traverse the maize-infecting fitness landscape towards the fitness peak occupied by extant MSV-A genotypes. It is plausible that such information may aid the elucidation of the actual history of MSV-A evolution, because other in vitro evolution studies have indicated that adaptive steps across fitness landscapes are constrained to relatively few, specific, evolutionary pathways [47-50].

Here we describe an improved version of the experimental system described by van der Walt et al. [45] that has enabled us to investigate the role played by host susceptibility, and the use of architecturally different pairs of defective starting chimaeras, in MSV evolution by recombination. In these experiments, we recovered recombinant genomes occupying a much wider variety of positions within the sequence space than those encountered in the original study, suggesting a plausible scenario for the initial adaptation of MSV to maize.

\section{Methods}

\section{Viruses}

Agro-infectious clones of wild-type MSV isolates MSVVW [51] and MSV-MatA [52], as well as the laboratoryconstructed reciprocal chimaeras of these viruses, MatMPCPVW and VWMPCPMat, and MatMPCPLIRVW and VWMPCPLIRMat [36] have been described previously. To explain the naming of the reciprocal chimaeras, the virus name following, say, the MP $+\mathrm{CP}$ segments in MatMPCPVW, indicates that the segments were derived from MSV-VW, whereas the rest of the MSV genome was derived from MSV-MatA (see Additional file 1). 


\section{Agro-infection and leafhopper transmissions}

We agro-inoculated 70 three-day-old seedlings of the MSV-sensitive maize genotype Sweetcorn cv. Golden Bantam (Millington Seed Co. USA), with a mixed inoculum of either MatMPCPVW+VWMPCPMat, or MatMPCPLIRVW+VWMPCPLIRMat as described by van der Walt et al. [45]. At approximately 30 days post inoculation (dpi) we transmitted viruses via leafhopper from each symptomatic plant to 13-day-old seedlings of the moderately MSV-resistant maize genotype PAN6099. For each transmission this was achieved by caging approximately eight Cicadulina mbila adults on a symptomatic Golden Bantam leaf for three days followed by transfer of cages to the third leaf of PAN6099 seedlings where they remained for the duration of the experiment $[45,53]$. We isolated DNA from symptomatic PAN6099 plants at approximately $30 \mathrm{dpi}$, and from symptomatic Golden Bantam plants at $60 \mathrm{dpi}$.

\section{Viral DNA isolation, cloning and sequencing}

Viral DNA was isolated from symptomatic leaves using the Extract-n-Amp ${ }^{\mathrm{TM}}$ Kit (Sigma-Aldrich), followed by rolling-circle amplification as previously described $[54,55]$. Amplified DNA was digested with the restriction enzyme BamHI to generate $\sim 2.7 \mathrm{~kb}$ monomeric MSV genomes which were gel-purified (GFX ${ }^{\mathrm{TM}}, \mathrm{GE}$ Healthcare), ligated into BamHI-digested pGEM ${ }^{\circledR}-3 Z f(+)$ (Promega Biotech) using T4 DNA ligase (Fermentas), and transformed into competent Escherichia coli (E. cloni $^{\circledR}$, Lucigen ${ }^{\circledR}$ Corporation) using standard protocols [56]. The resulting positive clones were sequenced using universal M13 forward and reverse sequencing primers and previously-described internal primers [57]. The genome sequences of an additional 11 recombinant viruses from van der Walt et al. [45] were included in our analyses.

These, along with the viruses obtained in this study, were named using informative details such as, sequentially, the maize cultivar used (either Golden Bantam, [GB], or Pan6099, [Pan]), which recombination experiment they were obtained from (the MatMPCPVW +VWMPCPMat and MatMPCPLIRVW+VWMPCPLIRMat co-infections being, respectively, experiment 1 and 2 ), and following a hyphen, whether the viruses sequenced were either recombinants or parental input virus (labelled as $\mathrm{R}$ and $\mathrm{I}$, respectively), and the specific plant number (and in a few cases followed also by the specific clone) from which the viruses were isolated.

\section{Construction of agro-infectious clones and fitness assays}

Infectious clones of recombinant viruses were constructed in pBI121 (Clontech Laboratories, USA) as previously described [58]. The fitness of these cloned recombinants, along with that of wt viruses MSV-MatA and MSV-VW and each of the parental artificial chimaeras, was assessed in the moderately MSV-resistant maize genotype Sweetcorn cv. STAR 7714 (Starke Ayres, South Africa) by quantifying the percentage chlorotic leaf area produced by the viruses on leaves 4 , 5 and 6 of symptomatically infected plants as previously described $[59,60]$.

More specifically, with the exclusion of the virus GB1$\mathrm{R} 2$, which was tested on approximately 42 separate plants, 18 of which became symptomatically infected, all the chlorotic areas caused by all of the viruses were assayed on leaves 4 through six for between 24 and 62 separate plants. Percentage chlorotic leaf areas caused by each virus on each plant were expressed as the mean (with $95 \%$ confidence interval) of the data obtained from leaves 4, 5 and 6 .

\section{Statistical analysis}

To test whether recombination breakpoints occurred more frequently in the coding or non-coding regions when using sensitive or resistant maize genotypes, we tallied the number of breakpoints occurring over the respective number of nucleotides (2219 nt for the coding regions, and $470 \mathrm{nt}$ for the non-coding regions) and calculated a two-tailed $\mathrm{p}$ value using the Fisher's exact test.

Similarly, using the percentage pair-wise difference between recombinant viruses and MSV-MatA - obtained using the different pairs of parental viruses and maize genotypes - we calculated a two-tailed $\mathrm{p}$ value using a Mann-Whitney test to test for differences in the overall genomic similarity of recombinant viruses to MSVMatA.

\section{Results and Discussion}

\section{Recombination efficiently generates maize-adapted}

progeny from maladapted parental MSV genomes

We investigated the adaptive value of recombination during mixed MSV infections by tracing the trajectory of evolution via recombination across a sequence space bounded by maize-adapted and non-maize-adapted wt MSV genotypes. Specifically, we used a variation of a previously described experimental system [45] in which defective, laboratory-constructed MSV recombinants collectively comprising the complete genomic sequence of a maize-adapted MSV isolate were co-inoculated into maize and allowed to recombine during a defined time period. We assessed the efficiency with which maize adapted progeny genomes were generated within this simple experimental system, with respect to two important factors: (1) the particular partitioning of maizeadaptive genetic polymorphisms within defective parental viruses, and (2) the differential selective challenges imposed by different maize genotypes. 
Seventy MSV-sensitive maize seedlings (Golden Bantam) were co-infected with each of the defective laboratory-constructed recombinant virus pairs MatMPCPVW + VWMPCPMat (containing reciprocal $m p$ and $c p$ exchanges between the maize-adapted MSV-A isolate, MSV-MatA, and the Digitaria-adapted MSV-B isolate, MSV-VW) and MatMPCPLIRVW+VWMPCPLIRMat (containing reciprocal $m p, c p$ and LIR exchanges). From each set of agro-infections we identified approximately 60 plants with symptoms (chlorotic streaking and stunting) that ranged from mild to severe. At 60 dpi we isolated and sequenced a single MSV genome from each symptomatic plant. In addition, we transmitted viruses using leafhoppers from each of 36 symptomatic plants inoculated with MatMPCPLIRVW+VWMPCPLIRMat and five symptomatic plants inoculated with MatMPCPVW+VWMPCPMat to individual thirteen-day old MSV-resistant plants (PAN6099) as previously done [45].

We observed symptomatic infections in 15 MSV-resistant maize plants that were infected via leafhopper with viruses derived from the MatMPCPLIRVW +VWMPCPLIRMat co-infections, and in one symptomatic MSV-resistant maize plant infected with viruses obtained from the MatMPCPVW+VWMPCPMat coinfections. Approximately 60 days after the co-inoculations into Golden Bantam and 30 days after the leafhopper transmissions, we isolated and sequenced single MSV genomes from each infected MSV-resistant plant. We also analysed a further 11 MatMPCPVW +VWMPCPMat derived recombinants arising in MSVresistant maize plants that were described by van der Walt et al. [45].

Complete genome sequences of the viruses isolated from symptomatic plants revealed that recombinant progeny arose frequently within the various mixed infections although this depended on the specific chimaeric nature of the co-inoculated viruses. While some of the viruses retrieved from MSV-sensitive maize were not recombinant, from the cohort of viruses transmitted via leafhoppers from sensitive to MSV-resistant maize all the isolated viruses were recombinant (Table 1; see also Additional file 2), presumably because of the greater selective pressures imposed by the resistant host, and/or due to a strong selective sieve during leafhopper transmission. As reported previously in co-inoculations with the MatMPCPVW+VWMPCPMat chimaera pair [45], we only retrieved parental viruses from some symptomatic plants. The parental viruses that were most frequently retrieved were those containing the maize adapted MSV-MatA derived $m p$ and $c p$ genes (i.e. VWMPCPLIRMat and VWMPCPMat). This was not surprising, since the $m p-c p$ module of MSV-MatA has been shown to be the primary pathogenicity determinant of MSV in maize [36]. The additional contribution of the LIR to MSV pathogenicity [30,36] was also corroborated here by the fact that the parental virus, VWMPCPLIRMat, differing from VWMPCPMat only by the presence of the MSV-MatA LIR, was retrieved twice as often from symptomatic plants (Table 1 ).

\section{Recombination breakpoint patterns are dependant on the starting parental chimaeras as well as the degree of host resistance}

In agreement with our previous findings [45], the recombinant viruses recovered from these experiments displayed between two and 22 recombination breakpoints (i.e. they were a mixture of simple and complex recombinants), with higher proportions of simple recombinants being isolated from MSV-sensitive plants than from MSV-resistant plants (Table 1).

Although the two sets of laboratory-constructed parental chimaeras differed with respect to the partitioning of maize-adapted MSV genetic material between their constituent genomes, the recombination breakpoint distributions detected within the progeny recombinants mirrored those seen in natural geminiviruses [13,46,61-63]. Specifically, the majority of recombinant viruses had recombination breakpoints in previously identified mastrevirus and/or begomovirus recombination hot-spots such as at the $c p /$ SIR interface and at the virion-strand origin of replication $(v$-ori) within the LIR $[13,44,61,64,65]$ (Figure 1). However, contrary to natural breakpoint distributions observed in mastreviruses and begomoviruses, fewer recombination breakpoints fell in the complementary-sense genes, and more fell within the $c p$ gene, particularly within the 3' half of the gene. Despite these differences between the natural and experimental recombination breakpoint distributions, both display a marked bias against recombination breakpoints within the protein-coding sequences, with the majority of cross-over events occurring in the intergenic regions ( $\mathrm{p}$-value $<0.0001$ for each individual data set, or combined data set). It is likely that these coding region cold-spots are at least partially attributable to selection against the disruptive effects that recombination within genes can have on amino acid interactions within the tertiary and/or quaternary structures of recombinant proteins [66].

Notwithstanding the similarities between the recombination breakpoint distributions observed in the different experiments, there are two potentially important differences between recombinants arising during the MatMPCPVW+VWMPCPMat and MatMPCPLIRVW +VWMPCPLIRMat co-infections. While the recombination breakpoints in the $5^{\prime}$ portion of $m p$ have previously been observed in field-isolated MSV recombinants [13] 
Table 1 Recombinant genomes arising during mixed infections of different chimaeric parental MSV genomes in differentially MSV-resistant maize genotypes.

\begin{tabular}{|c|c|c|c|c|c|c|}
\hline \multirow{2}{*}{$\begin{array}{c}\text { Input viruses } \\
\text { MatMPCPWW + WWMPCPMat }\end{array}$} & \multirow{2}{*}{$\begin{array}{c}\text { Maize } \\
\text { genotype }\end{array}$} & \multicolumn{2}{|c|}{ Parental virus ${ }^{\mathrm{b}}$} & \multirow{2}{*}{$\begin{array}{c}\begin{array}{c}\text { Recombinant } \\
\text { virus }^{\text {b }}\end{array} \\
77 \%(26)\end{array}$} & \multirow{2}{*}{$\begin{array}{c}\begin{array}{c}\text { Simple } \\
\text { recombinants }\end{array} \\
85 \%\end{array}$} & \multirow{2}{*}{$\begin{array}{c}\begin{array}{c}\text { Complex } \\
\text { recombinants }\end{array} \\
15 \%\end{array}$} \\
\hline & & $\begin{array}{c}3 \%(1) \\
\text { MatMPCPWW }\end{array}$ & $\begin{array}{c}21 \%(7), \\
\text { WWMPCPMat }\end{array}$ & & & \\
\hline & MSV-resistant & 0 & 0 & $100 \%(1)$ & $75 \%$ & $25 \%$ \\
\hline \multirow[t]{2}{*}{$\begin{array}{l}\text { MatMPCPLIRWW + } \\
\text { VWMPCPLIRMat }\end{array}$} & MSV-sensitive & 0 & $\begin{array}{c}45 \%(18) \\
\text { WWMPCPLIRMat }\end{array}$ & $55 \%(22)$ & $95 \%$ & $5 \%$ \\
\hline & MSV-resistant & 0 & $\begin{array}{c}7 \%(1), \\
\text { WWMPCPLIRMat }\end{array}$ & $93 \%(14)$ & $79 \%$ & $21 \%$ \\
\hline $\begin{array}{l}\text { MatMPCPWW + } \\
\text { WWMPCPMatt }^{a}\end{array}$ & MSV-resistant & $\begin{array}{c}8 \%(1) \\
\text { MatMPCPWW }\end{array}$ & $\begin{array}{c}42 \%(5) \\
\text { WWMPCPMat }\end{array}$ & $100 \%(11)$ & $73 \%$ & $27 \%$ \\
\hline
\end{tabular}

a Experimental results obtained by van der Walt et al. [45]

${ }^{b}$ Numbers in brackets indicate the total number of individual plants from which MSV genomes were isolated

and are not particularly unusual, in our experiments breakpoints were only observed within this region in coinfections initiated with the MatMPCPVW +VWMPCPMat chimaera pair. Martin and Rybicki [36] found genetic evidence of a possible $m p$-LIR interaction that might explain the selective advantage of recombination events in the $5^{\prime}$ portion of $m p$ that reunite LIR and $m p$ sequences derived from the maize-adapted MSVMatA isolate. Conversely, recombination breakpoints in this region during mixed infections of MatMPCPLIRVW and VWMPCPLIRMat would run the risk of separating MSV-MatA derived LIR and $m p$ sequences, thereby possibly disrupting previously detected DNA-DNA or DNA-protein LIR - $m p$ interactions (they have only
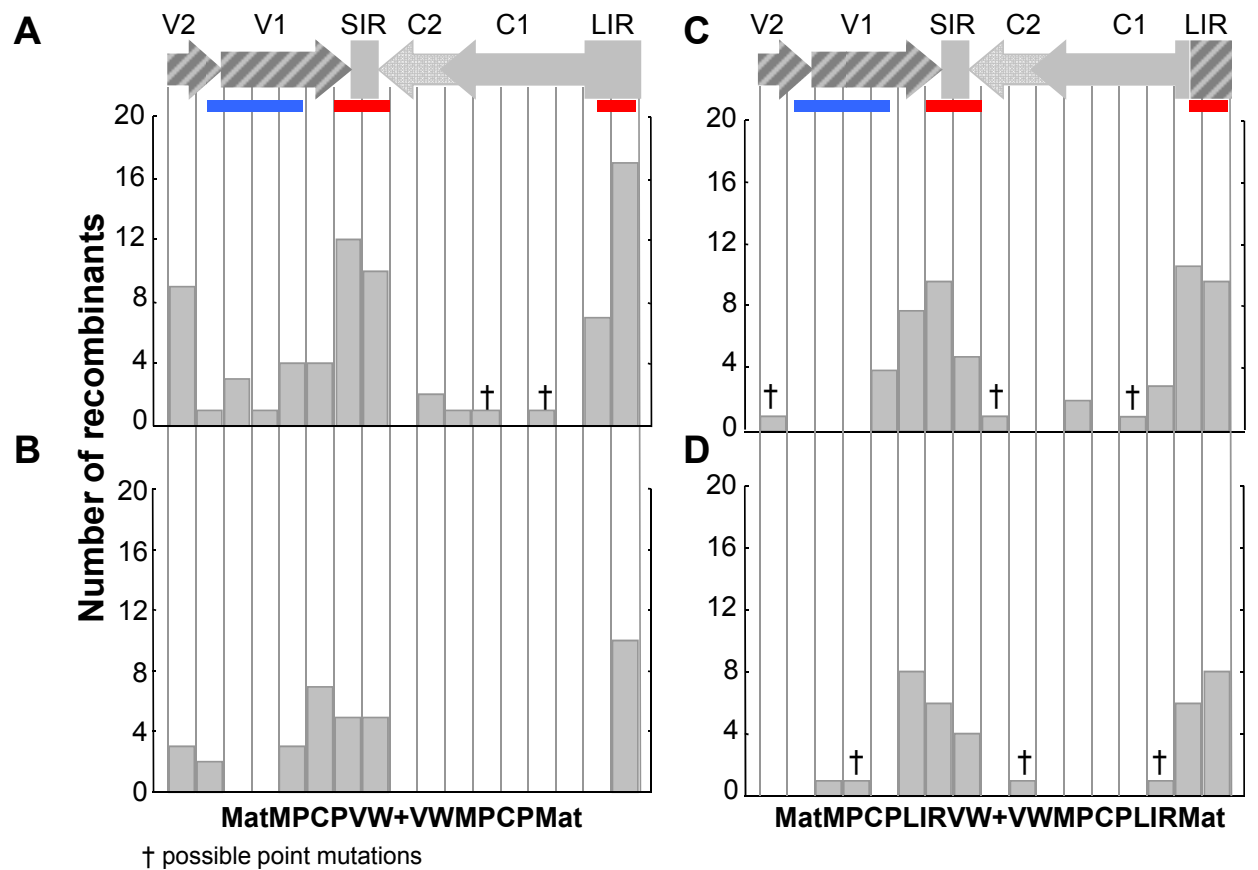

Figure 1 Genome-wide distribution of recombination breakpoints arising during different recombination experiments. A linearised MSV genome schematic divided into seventeen approximately equal segments. The light- and dark-grey hatched regions demarcate the regions swapped between the original wild-type viruses during construction of the chimaeric parental viruses (names indicated under each linearised genome). The total number of recombinants with breakpoints within each segment is represented using the bar graphs, where the recombinant viruses were obtained using the MatMPCPVW+WWMPCPMat chimaeric pair (panels A and B), or the MatMPCPLIRWW+WWMPCPLIRMat chimaeric pair (panels C and D) respectively in MSV-sensitive (panels A and C) and MSV-resistant (panels B and D) maize. The red and blue horizontal bars indicate, respectively, the approximate regions of prominent recombination hot-spots and cold-spots identified in wild-type MSV [13]. Genomic features: V2 = movement protein gene; V1 = coat protein gene; SIR = short intergenic region; C1/C2 = replication-associated protein gene; C1 = repA gene; $L I R=$ long intergenic region. 
been genetically detected and it is uncertain which of these interactions occur; [36]).

A second observation worth noting is that the MSVresistant and MSV-sensitive maize hosts gave rise to sets of recombinant viruses with different breakpoint distributions within the complementary-sense ORFs, C1 and $\mathrm{C} 2$. From the MSV-sensitive maize plants we identified six recombinants with breakpoints within this region (GB1-R18 and GB1-R19 in Figure 2A and GB2R3, GB2-R5, GB2-R7, GB2-R18 and GB2-R21_1 in Figure $2 \mathrm{C}$ ), while none were observed in this area of the recombinants isolated from the MSV-resistant maize. Two viruses obtained from resistant maize plants inoculated using leafhoppers previously fed on MatMPCPLIRVW+VWMPCPLIRMat co-infected plants, showed possible evidence of small recombination events in $\mathrm{C} 1 /$ C2 (Pan2-R4 and Pan2-R12, Figure 2D). However, these events would have only involved the exchange of a single polymorphic nucleotide and therefore could not be reliably distinguished from convergent point mutations. Although recombination within the $\mathrm{C} 1 / \mathrm{C} 2$ genes of field-isolated MSVs has been observed, the parental viruses shared greater than $\sim 95 \%$ sequence identity in all of these cases [13]. The presence of recombination breakpoints within this region in viruses that we isolated from sensitive maize plants implies that there is no biochemical impediment to recombination breakpoints falling in $\mathrm{C} 1 / \mathrm{C} 2$ when parental viruses are $<95 \%$ identical (in all cases here the parental viruses were $89 \%$ identical). Rather, the absence of breakpoints in this region in the resistant maize plants strongly implies that such recombinants are possibly usually defective, and that natural selection is responsible for their apparent rarity in nature.

Most of the recombinants produced during our experiments were essentially reconstructions of the original maize-adapted wt parental virus, MSV-MatA, with one breakpoint occurring within 200 nucleotides (nt) upstream or downstream of the $v$-ori, and another close to the $c p$ SIR interface (Figure 2). Importantly, and consistent with previous findings that the $v$-ori is a recombination hotspot $[13,37,44,64], 42 \%$ of all MatMPCPVW+VWMPCPMat recombinants $(10 / 26$ from the MSV-sensitive maize and 6/12 from the MSV-resistant maize) analysed in this study as well as $64 \%$ (7/11 from the MSV-resistant maize) analysed previously by van der Walt et al. [45], and 47\% of MatMPCPLIRVW+VWMPCPLIRMat derived recombinants (9/22 from MSV-sensitive maize and 8/14 from MSV-resistant maize) had a breakpoint within $14 \mathrm{nt}$ of this site (Figure 2).

Besides transfers of extensive contiguous sequences comprising nearly entire virion-sense or complementary-sense gene cassettes, many signals of transfers of much smaller fragments were also observed. While some of these may have represented the exchange of a single polymorphic nucleotide, it is difficult to distinguish such recombination events from possible convergent point mutations, and we therefore did not consider them any further with respect to our recombination analysis. Small recombination events entailing the exchange of two or more polymorphic nucleotides were not apparently clustered and occurred throughout the genome in the LIR (recombinants GB1-R23, Pan1-R11 and Pan1-R3), $c p$ (recombinants GB1-R1 and Pan1R11), $m p$ (recombinants GB1-R23, GB2-R18 and Pan1R2) and C1/C2 (recombinants GB1-18 and GB1-R19; see Figure $2 \mathrm{~A}$ and $2 \mathrm{~B}$ ).

Although almost all of the recombinants were unique to the plants from which they were isolated, occasionally identical recombinants were isolated from different plants suggesting that certain "recombinant solutions" were more easily accessible and/or selectively favoured. Examples of such recombinants derived from the MatMPCPVW+VWMPCPMat co-infections include GB1-R8 and GB1-R26 isolated from MSV-sensitive maize plants (Figure 2A), and Pan1-R8 and Pan1-R9 isolated from MSV-resistant maize plants (Figure 2B). Examples from the MatMPCPLIRVW+VWMPCPLIRMat co-infections include GB2-R6, GB2-R19, GB2-R12 and GB2-R21_2 isolated from MSV-sensitive maize (Figure 2C), and Pan2-R7 and Pan2-R8 or Pan2-R2 and Pan2-R3 (Figure 2C) isolated from MSV-resistant maize plants. Conversely, we also identified instances, exemplified by GB2-R21_1 and GB2-R21_2 (Figure 2C), where two different recombinants were isolated from the same plant.

\section{Recombinants tend to converge on the MSV-MatA genotype}

Given the potential importance of genetic recombination during evolutionary adaptation, we were interested in assessing the efficiency with which recombination could reassemble a genome resembling MSV-MatA - the easily accessible, and presumably "optimal", maize-adapted "target solution" genome that we used to construct the reciprocal parental chimaeras. In our experiments, recombination enabled the exploration of vast tracts of sequence space bounded by, in one dimension, the parental genomes used during the co-infections and, in a second dimension, the original wt viruses MSV-MatA and MSV-VW used to construct these parental genomes.

The simplest way in which all 248 MSV-MatA-derived polymorphisms carried by a set of reciprocal chimaeras could have recombined to form a single progeny genome was via two crossover events, at the junctions used to construct the parental chimaeric genomes. While the majority of the recombinant genomes appeared to 


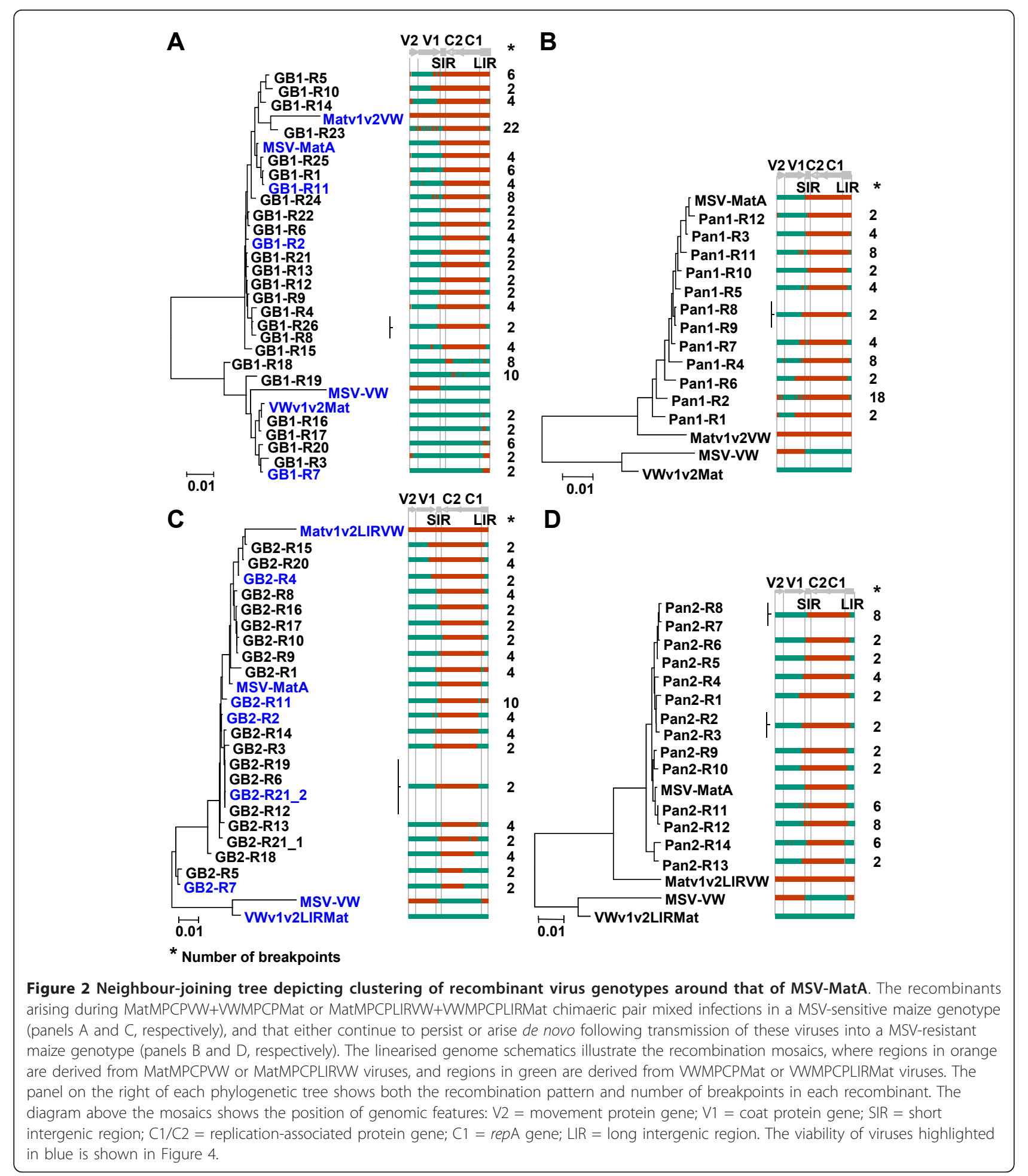

approximately represent such simple cross-over events, the degree to which progeny genomes recovered MSVMatA-derived polymorphisms varied according to the particular pair of reciprocal chimaeric viruses in the experiment, and with the type of host plant.
In recombinants recovered from MatMPCPVW +VWMPCPMat co-infections of the sensitive maize genotype, $31.5 \%-97.3 \%$ of polymorphic sites were identical to MSV-MatA. In contrast, recombinant progeny from the same reciprocal chimaeric parental genome pair 
isolated from resistant maize carried $88.3 \%-98.2 \%$ of the MSV-MatA polymorphisms.

The recombinants arising from MatMPCPLIRVW +VWMPCPLIRMat co-infections were generally more closely related to MSV-MatA than were recombinants isolated from MatMPCPVW+VWMPCPMat co-infections, with MSV-MatA contributing between 71.4\% 97.3\% of polymorphisms in recombinants isolated from MSV-sensitive maize, and between 92.9\% - 99.2\% of polymorphisms in recombinants isolated from resistant maize.

The differences in the extent to which recombinants in the four separate experiments converged on the MSV-MatA sequence is reflected in the conserved and consensus sequence polymorphism maps in Figure 3. Importantly, irrespective of the specific experimental conditions, only MSV-A-derived polymorphisms were absolutely conserved amongst all the recombinants obtained from each of the different experiments (see the conserved solution maps in Figure 3 indicating the origins of the invariant sites across all observed recombinants). Moreover, the consensus sequence of the recombinants (that is, the genome constructed from the most common polymorphisms observed at each variable site across all the observed recombinants) was between 93.69\% and $96.87 \%$ similar to the MSV-MatA sequence in all four of the experiments.

Whereas the recombinants isolated from MatMPCPVW+VWMPCPMat co-infections of MSVsensitive maize shared only a few conserved polymorphic sites around the 3' end of $m p$ and the 5' portion of $c p$ (Figure 3A), conserved sites amongst the recombinants from the MSV-resistant maize genotype additionally included all sites within $\mathrm{C} 1 / \mathrm{C} 2$ and many sites within the LIR and SIR regions (Figure 3B). In both host genotypes, the "conserved" regions of recombinants from MatMPCPLIRVW+VWMPCPLIRMat coinfections included sites in the 3' portion of $m p$, the 5' portion of $c p$, most of the $\mathrm{C} 2$ and the $\mathrm{C} 2$ proximal half of the SIR (Figure 3C and 3D). Recombinants obtained from resistant maize additionally contained MSV-A derived polymorphisms throughout most of their $\mathrm{C} 1$ and $\mathrm{C} 2$ ORFs and within the V2 proximal portion of their LIR sequences immediately downstream of the $v$ ori (Figure 3D).

Although the "consensus" recombinant progeny genomes arising from the different parental chimaera pairs in the two hosts were all remarkably MSV-MatA-like, some MSV-VW derived polymorphisms were invariably present around the sites used in the initial construction of the chimaeras from MSV-MatA and MSV-VW (Figure 3). Whereas in MatMPCPVW+VWMPCPMat derived recombinants the MSV-VW polymorphisms within the consensus occurred downstream of the $v$-ori, in the MatMPCPLIRVW+VWMPCPLIRMat derived recombinants they occurred upstream of this site. Although this pattern doubtlessly reflects the differences between the ligation sites used to construct the two reciprocal chimaeric pairs, it also indicates that the $v$-ori is a recombination hotspot.

\section{MSV-sensitive maize hosts provide a more permissive fitness landscape}

Although the "consensus" recombinant genomes generated under the different experimental conditions were very similar, there were notable differences in the relative ease with which recombination between the different co-infected parental chimaera pairs yielded recombinants that approximated MSV-MatA. For example, although the recombinants obtained from MSV-sensitive plants inoculated with MatMPCPVW +VWMPCPMat showed the most diverse recombination patterns, these recombinants were collectively not significantly less similar to MSV-MatA than those obtained from MSV-resistant plants, which displayed much less diverse patterns of recombination ( $p=0.57$, MannWhitney U-test; Figure 3A and 3B; see Additional file 3). However, recombinant viruses isolated from MSVsensitive plants co-infected with MatMPCPLIRVW +VWMPCPLIRMat were significantly less like MSVMatA than those obtained from MSV-resistant plants ( $p$ $=0.0014$, Mann-Whitney U-test; Figure 3C and 3D; see Additional file 3).

In the MSV-sensitive host, the recombinant viruses produced by each pair of chimaeric parental viruses were indistinguishable with respect to their similarity to MSV-MatA ( $\mathrm{p}=0.66$, Mann-Whitney U-test; see Additional file 3). In contrast, in MSV-resistant maize, the recombinants derived from MatMPCPLIRVW +VWMPCPLIRMat co-infections were collectively much more similar to MSV-MatA than their MatMPCPVW +VWMPCPMat-derived counterparts $(\mathrm{p}=0.0015$, Mann-Whitney U-test; see Additional file 3).

These results suggest that host susceptibility, as well as the configuration of parental genomes, can influence the efficiency with which fit genomes are assembled via recombination in a mixed infection. In other words, not only does a more selective maize host limit the possible trajectories of MSV evolution by reducing the diversity of arising recombinants, but it also is more selective of maize-adapted polymorphisms.

However, it remains unlikely that different degrees to which recombinants converge on the MSV-MatA sequence in co-infections of different parental chimaera pairs are attributable to selection alone. Rather, we expected that the parental chimaera pair that was assembled using cloning sites closest to the biochemically predisposed recombination hotspots, either within 

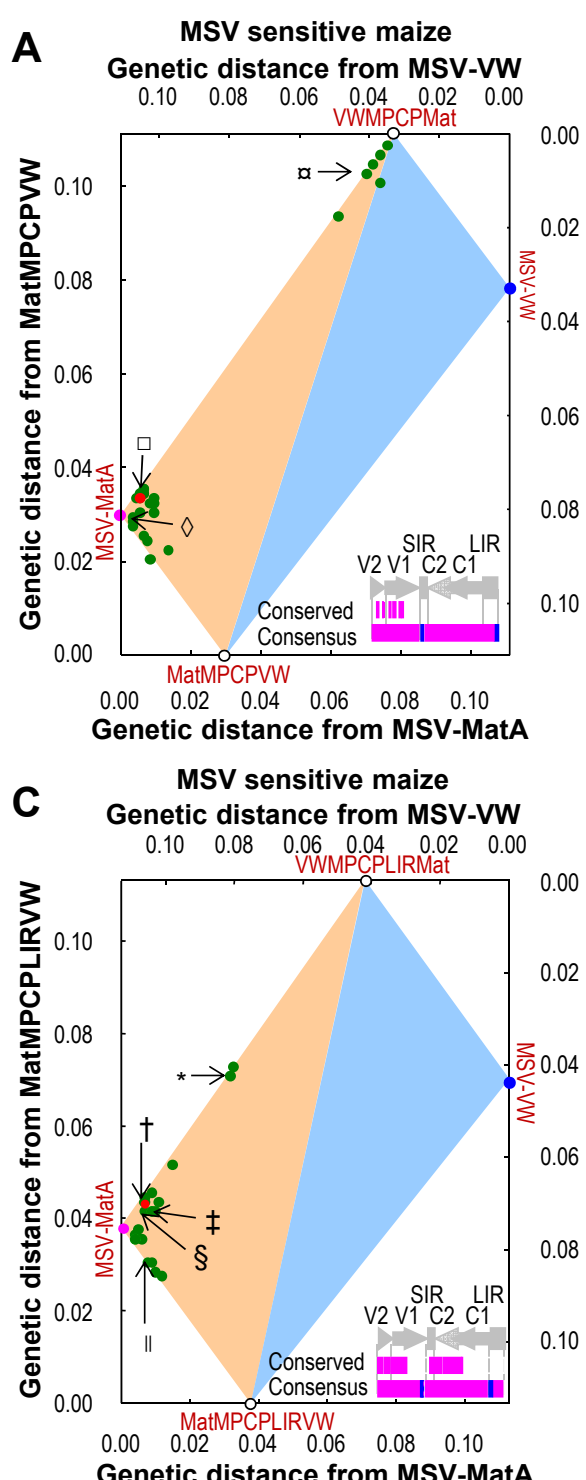

- Recombinant viruses

- Consensus solution

- Wild-type viruses

- Chimaeric parental viruses

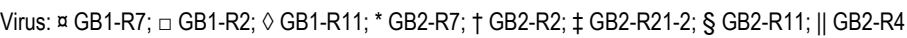

\section{B MSV resistant maize}

0.00

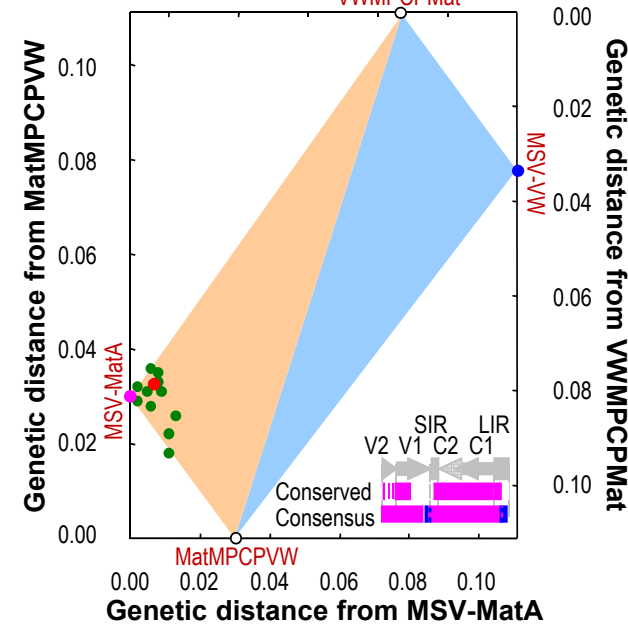

MSV resistant maize

D Genetic distance from MSV-VW $\begin{array}{llllll}0.10 & 0.08 & 0.06 & 0.04 & 0.02 & 0.00\end{array}$

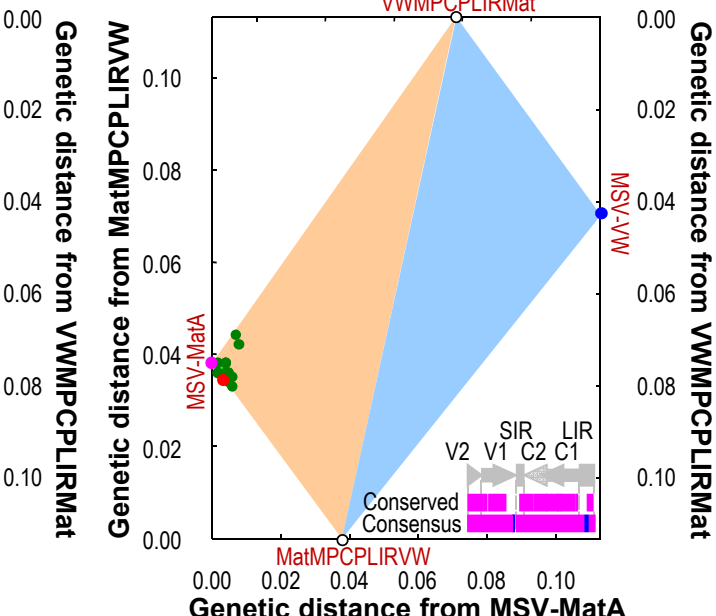

Figure 3 Distribution of recombinant viruses arising in maize within a two dimentional projection of the sequence space bounded by wild-type and chimaeric parental viruses. A Hamming distance graph showing the effects of maize genotype and chimaeric parental sequence pairs on how precisely the recombinant genotypes that arise during mixed infections converge on the target solution, MSV-MatA. Each green circle represents a single recombinant virus obtained using the MatMPCPWW+WWMPCPMat chimaeric pair in (A) MSV-sensitive and (B) MSV-resistant maize, and MatMPCPLIRVW+VWMPCPLIRMat chimaeric pair in (C) MSV-sensitive and (D) MSV-resistant maize. The red circle in each graph indicates the position of the consensus solution. The genome schematic embedded within each graph shows the regions in pink and blue derived from, respectively, MSV-MatA and MSV-WW. The conserved regions and overall consensus solutions are shown. The genome regions are as indicated in the legend of Figure 1. The viability of the viruses indicated using the symbols $a$ (GB1-R7), (GB1-R2), $\diamond$ (GB1-R11), * (GB2-R7), † (GB2-R2), ‡ (GB2-R21-2), § (GB2-R11), \|| (GB2-R4) is shown in Figure 4.

the LIR near the $v$-ori or within the SIR, would converge more easily on the ideal MSV-A solution. The reason for this is that recombination events at these sites would be most likely to reverse the steps used to construct the original parental chimaeras and to yield the wt maize- adapted genome (in this case MSV-MatA). This expectation was borne out by the observation that the cloning sites used to construct the MatMPCPLIRVW +VWMPCPLIRMat chimaera pair (the pair yielding recombinants that converged most closely on the MSV- 
MatA target solution) were on average $45 \mathrm{nt}$ closer to the biochemically predisposed recombination hotspots than those used to construct the MatMPCPVW +VWMPCPMat chimaera pair.

\section{The fitness of recombinant genomes}

The recombinant genomes that we recovered occupied a variety of positions within the sequence space separating MSV-MatA from MSV-VW (see Figure 3A and 3C). We hypothesised that MSV-MatA occupies a peak within the fitness landscape, and we therefore compared the fitness in maize of eight of the recombinants recovered from our experiments with that of MSV-MatA, MSVVW and their parental reciprocal chimaeras. As an approximate measure of viral fitness, we agroinoculated a moderately MSV-resistant maize genotype (Sweetcorn cV. STAR 7714) and quantified the percentage chlorotic leaf areas on leaves 4, 5 and 6 of successfully infected plants. The selection of this genotype was due to it being amenable to producing more discriminative infection data, than either the MSV-sensitive genotype within which most of the tested viruses produced indistinguishable symptoms, or the MSV-resistant genotype within which some of the viruses produced no symptoms at all. Although increased pathogenicity does not necessarily equate with increased fitness in nature, in the context of MSV infecting individual maize plants, replicative fitness and pathogenicity seem to be quite highly correlated $(43,52)$.

All of the recombinants from MatMPCPVW +VWMPCPMat co-infections, namely GB1-R2, GB1-R7 and GB1-R11, produced symptoms that were less severe than those of VWMPCPMat, the more virulent of the two parental chimaeras. One recombinant, GB1-R7, was even less severe than MatMPCPVW - the least virulent of the two parental chimaeras - but was nevertheless slightly more severe than MSV-VW (Figure 4A). A comparison of the mean percentage chlorotic leaf area produced by the three recombinants suggests that symptom severity correlated with genetic distance from the MSVMatA genomic sequence. Thus, of the three recombinants, GB1-R7 was the least like MSV-A (only 36.9\% of the $248 \mathrm{MSV}-\mathrm{MatA} / \mathrm{MSV}-\mathrm{VW}$ polymorphic nucleotides were derived from MSV-MatA), and was also the least virulent. GB1-R2, which derived $94.6 \%$ of its polymorphic nucleotides from MSV-A, was slightly more virulent, and GB1-R11 was the most genetically similar to MSV-A (97.3\% of the polymorphic nucleotides are from MSV-MatA) and produced the most severe symptoms. While these data suggest an apparent trend, one should note both that the $95 \%$ confidence intervals (CI) of the mean symptom severity estimates of these recombinants overlap extensively (Figure 4A) and that the correlation between genetic distance from MSV-MatA and symptom severity is not statistically supported $(\mathrm{p}=0.43$; Spearman ranks test).

Of the five recombinants derived from MatMPCPLIRVW+VWMPCPLIRMat co-infections, four (GB2R2, GB2-R4, GB2-R7, and GB2-R11) produced symptoms as severe as MSV-MatA, but one (GB2-R21_2) was only slightly more severe than MSV-VW and MatMPCPLIRVW (Figure 4B). GB2-R7 was the least MSV-A-like of these recombinants $(72.3 \%$ of the polymorphic sites were derived from MSV-MatA) and accordingly produced less severe symptoms than did the more MSVMatA-like recombinants GB2-R4, GB2-R11, and GB2R2 (which respectively obtained 93.8\%, 94.6\%, and 94.6\% of their polymorphic sites from MSV-MatA). However, the $95 \%$ CI of the chlorotic leaf area measurements made for these viruses were all largely overlapping (Figure 4B) and they probably do not have very different degrees of pathogenicity in maize. It is noteworthy that in addition to being clustered closer to the ideal solution, MSV-MatA, the MatMPCPLIRVW+VWMPCPLIRMat derived recombinants were also on average substantially fitter than those derived using the MatMPCPVW+VWMPCPMat chimaera pair (Figure 4A and 4B; $\mathrm{p}=0.064$ Mann Whitney U-test).

Although GB2-R21_2 was more MSV-A-like than GB2-R7 (respectively $92.9 \%$ and $72.3 \%$ of polymorphic sites derived from MSV-MatA), the symptoms it produced in maize were significantly less severe than those produced by all of the other recombinants tested. It is noteworthy however, that GB2-R21_2 carries a mutation in the C1 ORF that differentiates it from both MSV-VW and MSV-MatA. Although this mutation is silent for both Rep and RepA, it could conceivably have an adverse effect on ssDNA genomic or transcribed RNA secondary structures, as has been demonstrated for other silent Rep mutations $[67,68]$.

Although of low resolution, our survey of the fitness landscape surrounding the maize-adapted MSV-MatA genotype and the non-maize-adapted MSV-VW genotype in the maize cultivar Sweetcorn (STAR 7714) suggests a feasible evolutionary trajectory leading to the maize-adapted MSV-A strain (represented here by MSV-MatA[52]). Being mildly symptomatic in MSVsensitive maize genotypes $[60,61,65,69]$, the Digitariaadapted MSV-B (represented here by MSV-VW), -G and -F strains probably occupy the 'lowlands' of the "MSV in maize" fitness landscape. In addition to natural examples [13], our experiments demonstrate that recombination between these strains resulting in the exchange of a maize-specific pathogenicity determinant, the $m p$-cp gene module [36], could have greatly improved the fitness of the ancestral MSV-A virus. Even in our most permissive maize genotype, large portions of the maize-adapted MSV-MatA-derived $m p-c p$ 


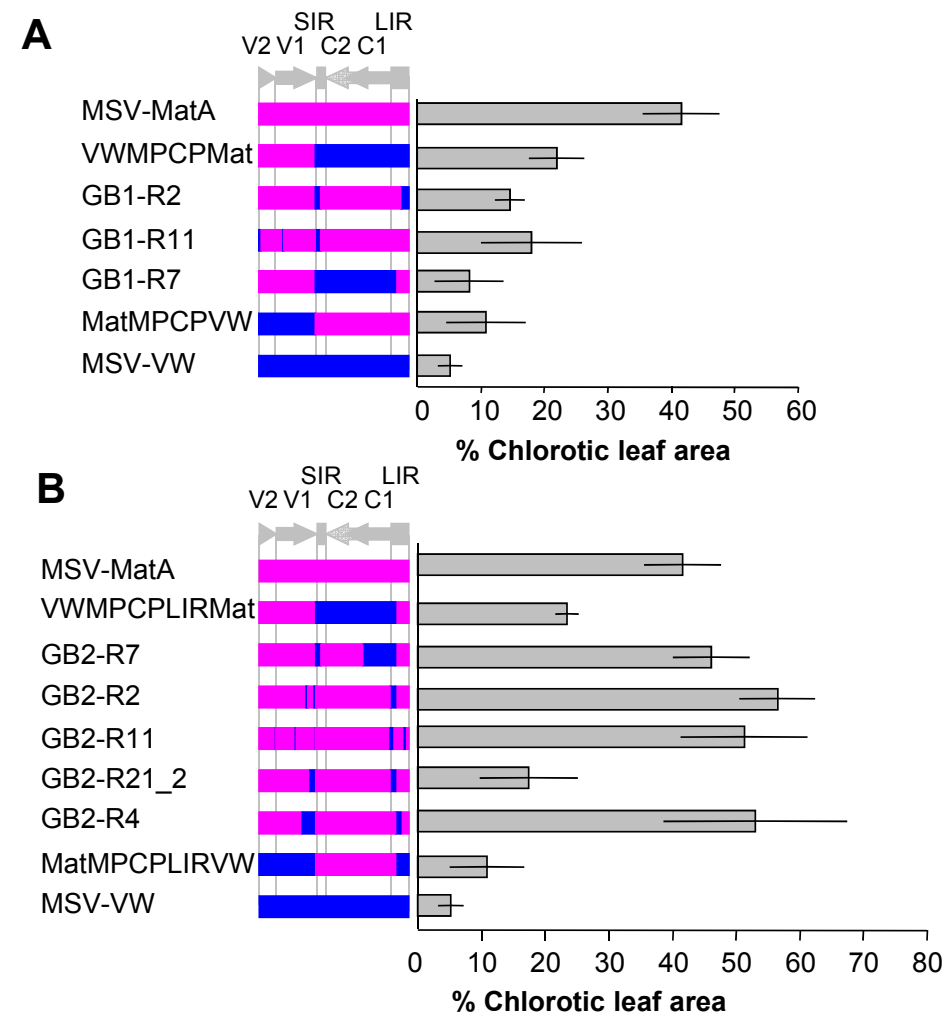

Figure 4 Fitness assay of parental MSV genomes and recombinant MSV viruses. The viability of recombinant viruses compared to that of their chimaeric parents, and the wild-type viruses MSV-MatA and MSV-WW. Recombinants recovered from MSV-sensitive plants (cv. Golden Bantam) after agroinoculation with the pair MatMPCPWW+WWMPCPMat (A) and MatMPCPLIRWW+WWMPCPLIRMat (B) were tested in moderatelyresistant maize (CV. STAR 7714). Mean chlorotic leaf areas (and the $95 \%$ confidence intervals of these estimates indicated by error bars) observed on leaves 4, 5 and 6 of symptomatic plants. Shown in blue and pink on the genome schematic are regions derived from MSV-VW and MSVMatA, respectively. The genome regions are as indicated in the legend of Figure 1.

sequence were conserved across all the recombinants. From this point onwards, as is implied by the relative fitness value of all the recombinants we tested, our results suggest that further exploration of the fitness landscape in permissive maize genotypes, either by further recombination or by point mutation, could have enabled a prototypical recombinant MSV-A to progressively climb higher on the fitness landscape to eventually attain the altitudes that have been reached by MSV-A genotypes found today throughout Africa [70].

\section{Conclusions}

Using an established model system for analysing the evolution and adaptation of MSV to maize plants, we demonstrate that despite diverse recombinants emerging during mixed infections involving two separate sets of parental viruses and two different hosts, both the overall distributions of recombination breakpoints and the average patterns of recombination are remarkably similar across all experimental conditions. Most notably, in all experiments the consensus of all observed recombinants deterministically converged upon that of the maize-adapted MSV-A genotype, MSV-MatA, which was initially used to construct the parental chimaera pairs. Besides converging on the MSV-MatA sequence, when tested in isolation some of the recombinants also produced symptoms in maize that approached those produced by MSV-MatA.

It is clear from our study that the biochemically predisposed recombination hot-spots within the MSV genome strongly influenced the recombination patterns that we observed in our experiments. It is also evident that varying mechanistic predispositions to recombination across the MSV genome can constrain the efficiency with which recombination provides access to fitness peaks within the sequence space. However, it is noteworthy that we have provided evidence in MSV that, as is becoming apparent in other viruses such as Human immunodeficiency virus [71], recombination hotspots within the intergenic regions correspond with genome sites at which recombination breakpoints are likely to have the smallest deleterious impact on virus viability - 
a finding which suggests that the MSV genome may have specifically evolved to accommodate a recombinogenic life-style.

Finally, our results indicate a plausible scenario for the creation and early evolution of the maize adapted MSVA strain through recombination between two Digitariaadapted MSVs. The complex recombination patterns that we have sometimes observed indicate that within a permissive MSV-sensitive maize genotype, the prototypical MSV-A genome could conceivably have been assembled through a series of adaptive recombination events (and possibly also compensatory and/or adaptive point mutations) that incrementally nudged it towards the fitness peak that MSV-A currently populates in maize.

\section{Additional material}

Additional file 1: Genome organization of wild-type and chimaeric MSV genomes used in this study. The curved arrows indicate open reading frames (ORFs) diverging from the long intergenic region (LIR) and eventually converging on the short intergenic region (SIR). The intergenic regions, the ORFs in the complementary-sense - which encode the replication-associated protein (Rep) and the replicationassociated protein (RepA) - and the ORFs in the virion-sense - which encode the movement protein (MP) and the coat protein (CP) - are colored red (in the case of MSV-WW) or blue (in the case of MSV-MatA). This color-code is also used to delineate the genomic portions of MSVMatA and MSV-WW used to construct the pair of reciprocal chimaeric MSV genomes used to conduct recombination experiments.

Additional file 2: Alignment of wild-type and recombinant MSV genomes. Full genome sequence alignment of MSV viruses aligned from the start codon of the movement protein gene. The wild-type viruses MSV-MatA and MSV-VW, as well as the chimaeric parental viruses and recombinants used in two different recombination experiments are included. The file is in FASTA format and should be viewed using a sequence analysis program such as Mega5.

Additional file 3: Pair-wise distance of recombinant viruses from MSV-MatA. Distribution of recombinant viruses using percentage pairwise distance and statistical analysis (Mann-Whitney $\mathrm{U}$ test, two-tailed $\mathrm{p}$ value) of the approximation of each group of viruses to MSV-MatA. Recombinant viruses were obtained using different pairs of parental chimaeric MSV genomes, inoculated into differentially-resistant maize genotypes.

\section{Acknowledgements}

We would like to thank Dr. Dionne N. Shepherd for constructive criticisms and for offering to review and edit the manuscript. This project was funded by the National Research Foundation of South Africa. ALM was supported by the University of Cape Town, the Canon Collins Trust and the National Research Foundation (South Africa). AV was supported by the Carnegie Corporation of New York. DPM was supported by the Wellcome Trust.

\section{Author details}

'Department of Molecular and Cell Biology, University of Cape Town, Rondebosch, 7701, Cape Town, South Africa. ${ }^{2}$ Kapa Biosystems, P.O. Box 12961, Mowbray, 7705, South Africa. ${ }^{3}$ Biomolecular Interaction Centre, University of Canterbury, Private Bag 4800, Christchurch, 8140, New Zealand. ${ }^{4}$ School of Biological Sciences, University of Canterbury, Private Bag 4800, Christchurch, 8140, New Zealand. ${ }^{5}$ Electron Microscope Unit, University of Cape Town, Rondebosch, 7701, Cape Town, South Africa. ${ }^{6}$ Computational Biology Group, Institute of Infectious Disease and Molecular Medicine,
University of Cape Town, Observatory, 7925, Cape Town, South Africa. ${ }^{7}$ Centre for High-Performance Computing, Rosebank, Cape Town, South Africa.

\section{Authors' contributions}

ALM, EvdW, DPM, EPR conceived the study and participated in its design. ALM, DPM, AV isolated, cloned and sequenced the viral genomes and performed basic viral bioinformatics. ALM, DPM carried out plant inoculations and virulence assays, analyzed and interpreted the data. ALM, DPM, EvdW, AV, EPR drafted and revised the manuscript. All authors read and approved the final manuscript.

Received: 15 September 2011 Accepted: 2 December 2011

Published: 2 December 2011

\section{References}

1. Duffy S, Holmes EC: Validation of high rates of nucleotide substitution in geminiviruses: phylogenetic evidence from East African cassava mosaic viruses. J Gen Virol 2009, 90:1539-1547.

2. van der Walt E, Martin DP, Varsani A, Polston JE, Rybicki EP: Experimental observations of rapid Maize streak virus evolution reveal a strandspecific nucleotide substitution bias. Virol J 2008, 5:104

3. Harkins GW, Martin DP, Duffy S, Monjane AL, Shepherd DN, Windram OP, Owor BE, Donaldson L, van Antwerpen T, Sayed RA, Flett B, Ramusi M, Rybicki EP, Peterschmitt M, Varsani A: Dating the origins of the maizeadapted strain of maize streak virus, MSV-A. J Gen Virol 2009, 90(12):3066-3074.

4. Grigoras I, Timchenko T, Grande-Perez A, Katul L, Vetten HJ, Gronenborn B: High variability and rapid evolution of a nanovirus. J Virol 2010, 84:9105-9117.

5. Sanjuán R, Nebot MR, Chirico N, Mansky LM, Belshaw R: Viral mutation rates. J Virol 2010, 84(19):9733-9748.

6. Chetverin AB: The puzzle of RNA recombination. FEBS Lett 1999, 460:1-5.

7. Idris AM, Mills-Lujan K, Martin K, Brown JK, Melon Chlorotic Leaf Curl Virus: Characterization and differential reassortment with closest relatives reveal adaptive virulence in the Squash Leaf Curl Virus clade and host shifting by the host-restricted Bean Calico Mosaic Virus. J Virol 2008, 82(4):1959-1967.

8. Jeske H: Geminiviruses. Curr Top Microbiol Immunol 2009, 331:185-226.

9. Domingo E: Mechanisms of viral emergence. Vet Res 2010, 41(6):38.

10. Christman MC, Kedwaii A, Xu J, Donis RO, Lu G: Pandemic (H1N1) 2009 virus revisited: An evolutionary retrospective. Infect Genet Evol 2011, 11(5):803-811.

11. Lauring AS, Andino R: Quasispecies theory and the behaviour of RNA viruses. PLOS Pathog 2010, 6(7):e1001005.

12. Lorenzo-Redondo R, Bordería AV, Lopez-Galindez C: Dynamics of in vitro fitness recovery of HIV-1. J Virol 2011, 85(4):1861-1870.

13. Varsani A, Shepherd DN, Monjane AL, Owor BE, Erdmann JB, Rybicki EP, Peterschmitt M, Briddon RW, Markham PG, Oluwafemi S, Windram OP, Lefeuvre P, Lett J-M, Martin DP: Recombination, decreased host specificity and increased mobility may have driven the emergence of maize streak virus as an agricultural pathogen. J Gen Virol 2008, 89:2063-2074.

14. Patil BL, Fauquet CM: Cassava mosaic geminiviruses: actual knowledge and perspectives. Mol Plant Pathol 2009, 10(5):685-701.

15. Briddon RW, Bedford ID, Tsai JH, Markham PG: Analysis of the nucleotide sequence of the treehopper-transmitted geminivirus, tomato pseudocurly top virus, suggests a recombinant origin. Virology 1996, 219:387-394.

16. Varsani A, Shepherd DN, Dent K, Monjane AL, Rybicki EP, Martin DP: A highly divergent South African geminivirus species illuminates the ancient evolutionary history of this family. Virol J 2009, 25(6):36.

17. Gibbs MJ, Weiller GF: Evidence that a plant virus switched hosts to infect a vertebrate and then recombined with a vertebrate-infecting virus. $P$ Natl Acad Sci USA 1999, 96:8022-8027.

18. Ravantti JJ, Bamford DH: Geminiviruses: A tale of a plasmid becoming a virus. BMC Evol Biol 2009, 9:112.

19. Bosque-Pérez NA: Eight decades of Maize streak virus research. Virus Research 2000, 71:107-121.

20. Martin DP, Shepherd DN: The epidemiology, economic impact and control of maize streak disease. Food Security 2009, 1:305-315. 
21. Shepherd DN, Martin DP, van der Walt E, Dent K, Varsani A, Rybicki EP. Maize streak virus: an old and complex 'emerging' pathogen. Mol Plant Pathol 2010, 11:1-12.

22. Boulton MI, Buchholz WG, Marks MS, Markham PG, Davies JW: Specificity of Agrobacterium-mediated delivery of maize streak virus DNA to members of the Gramineae. Plant Mol Biol 1989, 12:31-40.

23. Lazarowitz SG, Pinder AJ, Damsteegt VD, Rogers SG: Maize streak virus genes essential for systemic spread and symptom development. EMBO J 1989, 8:1023-1032.

24. Woolston CJ, Reynolds HV, Stacey NJ, Mullineaux PM: Replication of Wheat dwarf virus DNA in protoplasts and analysis of coat protein mutants in protoplasts and plants. Nucleic Acids Res 1989, 17:6029-6041.

25. Lazarowitz SG, Pinder AJ: Molecular genetics of Maize streak virus. UCLA Sym BI 1989, 101:167-183.

26. Hofer JM, Dekker EL, Reynolds HV, Woolston CJ, Cox BS, Mullineaux PM: Coordinate regulation of replication and virion sense gene expression in wheat dwarf virus. Plant Cell 1992, 4:213-223.

27. Collin S, Fernández-Lobato M, Gooding PS, Mullineaux PM, Fenoll C: The two nonstructural proteins from wheat dwarf virus involved in viral gene expression and replication are retinoblastoma-binding proteins. Virology 1996, 219:324-329.

28. Zhan X, Richardson KA, Haley A, Morris BA: The activity of the coat protein promoter of chloris striate mosaic virus is enhanced by its own and C1C2 gene products. Virology 1993, 193(1):498-502.

29. Hefferon KL, Moon YS, Fan Y: Multi-tasking of nonstructural gene products is required for bean yellow dwarf geminivirus transcriptional regulation. FEBS J 2006, 273(19):4482-94.

30. Willment JA, Martin DP, Palmer KE, Schnippenkoetter WH, Shepherd DN, Rybicki EP: Identification of long intergenic region sequences involved in maize streak virus replication. J Gen Virol 2007, 88:1831-1841.

31. Gabriel G, Dauber B, Wolff T, Planz O, Klenk H-D, Stech J: The viral polymerase mediates adaptation of an avian influenza virus to a mammalian host. P Natl Acad Sci USA 2005, 102(5):18590-18595.

32. Traoré $O$, Pinel-Galzi A, Issaka $S$, Poulicard N, Aribi J, Aké $S$, Ghesquière A, Séré $Y$, Konaté $G$, Hébrard E, Fargette D: The adaptation of Rice yellow mottle virus to the elF(iso)4G-mediated rice resistance. Virology 2010, 408:103-108.

33. Hajimorad MR, Wen R-H, Eggenberger AL, Hill JH, Saghai Maroof MA: Experimental adaptation of an RNA virus mimics natural evolution. $J$ Virol 2011, 85(6):2557-2564

34. Briddon RW, Pinner MS, Stanley J, Markham PG: Geminivirus coat protein gene replacement alters insect specificity. Virology 1990, 177(1):85-94.

35. Liu L, Pinner MS, Davies JW, Stanley J: Adaptation of the geminivirus bean yellow dwarf virus to dicotyledonous hosts involves both virion-sense and complementary-sense genes. J Gen Virol 1999, 80:501-6.

36. Martin DP, Rybicki EP: Investigation of maize streak virus pathogenicity determinants using chimaeric genomes. Virology 2002, 300:180-188.

37. Schnippenkoetter WH, Martin DP, Willment JA, Rybicki EP: Forced recombination between distinct strains of Maize streak virus. $J$ Gen Virol 2001, 82:3081-3090.

38. Isnard M, Granier M, Frutos R, Reynaud B, Peterschmitt M: Quasispecies nature of three maize streak virus isolates obtained through different modes of selection from a population used to assess response to infection of maize cultivars. J Gen Virol 1998, 79(12):3091-9.

39. Ge LM, Zhang JT, Zhou XP, Li HY: Genetic structure and population variability of Tomato yellow leaf curl China virus. J Virol 2007, 81:5902-5907

40. Urbino C, Thébaud G, Granier M, Blanc S, Peterschmitt M: A novel cloning strategy for isolating, genotyping and phenotyping genetic variants of geminiviruses. Virol J 2008, 31(5):135.

41. Harkins GW, Delport W, Duffy S, Wood N, Monjane AL, Owor BE, Donaldson L, Saumtally S, Triton G, Briddon RW, Shepherd DN, Rybicki EP, Martin DP, Varsani A: Experimental evidence indicating that mastreviruses probably did not co-diverge with their hosts. Virol J 2009, 6:104.

42. Jeske $H$, Lütgemeier M, Preiss W: DNA forms indicate rolling circle and recombination-dependent replication of Abutilon mosaic virus. EMBO J 2001, 20(21):6158-67.

43. Martin DP, van der Walt E, Posada D, Rybicki EP: The evolutionary value of recombination is constrained by genome modularity. PLOS Genet 2005, 1(4):e51.
44. García-André S, Tomas DM, Sanchez-Campos S, Navas-Castillo J, Moriones E: Frequent occurrence of recombinants in mixed infections of tomato yellow leaf curl disease-associated begomoviruses. Virology 2007, 365:210-219.

45. van der Walt E, Rybicki EP, Varsani A, Polston JE, Billharz R, Donaldson L, Monjane AL, Martin DP: Rapid host adaptation by extensive recombination. J Gen Virol 2009, 90:734-746.

46. Owor BE, Martin DP, Shepherd DN, Edema R, Monjane AL, Rybicki EP, Thomson JA, Varsani A: Genetic analysis of maize streak virus isolates from Uganda reveals widespread distribution of a recombinant variant. J Gen Virol 2007, 88:3154-3165.

47. Hayashi Y, Aita T, Toyota H, Husimi Y, Urabe I, Yomo T: Experimental rugged fitness landscape in protein sequence space. PLOS ONE 2006, 1(1):e96.

48. Weinreich DM, Delaney NF, DePristo MA, Hartl DL: Darwinian evolution can follow only very few mutational paths to fitter proteins. Science 2006, 312:111-114.

49. Lozovsky ER, Chookajorn T, Brown KM, Imwong M, Shaw PJ, Kamchonwongpaisan S, Neafsey DE, Weinreich DM, Hartl DL: Stepwise acquisition of pyrimethamine resistance in the malaria parasite. $P$ Natl Acad Sci USA 2009, 106:12025-12030.

50. Ohshima K, Akaishi S, Kajiyama H, Koga R, Gibbs AJ: Evolutionary trajectory of turnip mosaic virus populations adapting to a new host. J Gen Virol 2010, 91:788-801.

51. Willment JA, Martin DP, van der Walt E, Rybicki EP: Biological and genomic sequence characterization of Maize streak virus isolates from wheat. Phytopathology 2002, 92:81-86.

52. Martin DP, Willment JA, Billharz R, Velders R, Odhiambo B, Njuguna J, James D, Rybicki EP: Sequence diversity and virulence in Zea mays of Maize streak virus isolates. Virology 2001, 288:247-255.

53. Shepherd DN, Mangwende T, Martin DP, Bezuidenhout M, Thomson JA, Rybicki EP: Inhibition of maize streak virus (MSV) replication by transient and transgenic expression of MSV replication-associated protein mutants. J Gen Virol 2007, 88:325-336.

54. Inoue-Nagata AK, Albuquerque LC, Rocha WB, Nagata T: A simple method for cloning the complete begomovirus genome using the bacteriophage Q29 DNA polymerase. J Virol Methods 2004, 116:209-211.

55. Shepherd DN, Martin DP, Lefeuvre P, Monjane AL, Owor BE, Rybicki EP, Varsani A: A protocol for the rapid isolation of full geminivirus genomes from dried plant tissue. J Virol Methods 2008, 149:97-102.

56. Sambrook J, Fritsch EF, Maniatis T: Molecular cloning: A laboratory manual. Cold Spring Harbor, NY: Cold Spring Harbor Laboratory; 21989.

57. Owor BE, Shepherd DN, Taylor NJ, Edema R, Monjane AL, Thomson JA, Martin DP, Varsani A: Successful application of FTA Classic Card technology and use of bacteriophage Q29 DNA polymerase for largescale field sampling and cloning of complete maize streak virus genomes. J Virol Methods 2007, 140:100-105.

58. Martin DP, Rybicki EP: Improved efficiency of Zea mays agroinoculation with Maize streak virus. Plant Dis 2000, 84:1096-1098.

59. Martin DP, Rybicki EP: Microcomputer-based quantification of maize streak virus symptoms in Zea mays. Phytopathology 1998, 88:422-427.

60. Martin DP, Willment JA, Rybicki EP: Evaluation of Maize streak virus pathogenicity in differentially resistant Zea mays genotypes. Phytopathology 1999, 89:695-700.

61. Lefeuvre P, Martin DP, Hoareau M, Naze F, Delatte $H$, Thierry M, Varsani A, Becker N, Reynaud B, Lett J-M: Begomovirus 'melting pot' in the southwest Indian Ocean islands: molecular diversity and evolution through recombination. J Gen Virol 2007, 88:3458-3468.

62. Martin DP, Briddon RW, Varsani A: Recombination patterns in dicotinfecting mastreviruses mirror those found in monocot-infecting mastreviruses. Arch Virol 2011, 156(8):1463-1469.

63. Pita JS, Fondong VN, Sangaré A, Otim-Nape GW, Ogwal S, Fauquet CM: Recombination, pseudorecombination and synergism of geminiviruses are determinant keys to the epidemic of severe cassava mosaic disease in Uganda. J Gen Virol 2001, 82:655-665.

64. Stenger DC, Revington GN, Stevenson MC, Bisaro DM: Replicational release of geminivirus genomes from tandemly repeated copies: evidence for rolling-circle replication of a plant viral DNA. P Natl Acad Sci USA 1991, 88:8029-8033. 
65. Schnippenkoetter WH, Martin DP, Hughes F, Fyvie M, Willment JA, James D, von Wechmar B, Rybicki EP: The biological and genomic characterisation of three mastreviruses. Arch Virol 2001, 146:1075-1088.

66. Lefeuvre P, Lett JM, Reynaud B, Martin DP: Avoidance of protein fold disruption in natural virus recombinants. PLoS Pathog 2007, 3(11):e181.

67. Boulton MI, King DI, Donson J, Davies JW: Point substitution in a promoter-like region and the V1 gene affect the host range and symptoms of maize streak virus. Virology 1991, 183(1):114-121.

68. Shepherd DN, Martin DP, Varsani A, Thomson JA, Rybicki EP, Klump HH: Restoration of native folding of single-stranded DNA sequences through reverse mutations: An indication of a new epigenetic mechanism. Arch Biochem Biophys 2006, 453:108-122.

69. Pinner MS, Markham PG, Markham RH, Dekker EL: Characterisation of maize streak virus - description of strains - symptoms. Plant Pathol 1988, 37:74-87.

70. Monjane AL, Harkins GW, Martin DP, Lemey P, Lefeuvre P, Shepherd DN, Oluwafemi S, Simuyandi M, Zinga I, Komba EK, Lakoutene DP, Mandakombo N, Mboukoulida J, Semballa S, Tagne A, Tiendrébéogo F, Erdmann JB, van Antwerpen T, Owor BE, Flett B, Ramusi M, Windram OP, Syed R, Lett J-M, Briddon RW, Markham PG, Rybicki EP, Varsani A:

Reconstructing the history of Maize streak virus strain A dispersal to reveal diversification hot spots and its origin in southern Africa. J Virol 2011, 85(18):9623-9636.

71. Simon-Loriere E, Martin DP, Weeks KM, Negroni M: RNA structures facilitate recombination mediated gene swapping in HIV-1. J Virol 2010, 84(24):12675-12682.

\section{doi:10.1186/1471-2148-11-350}

Cite this article as: Monjane et al:: Recombination hotspots and host susceptibility modulate the adaptive value of recombination during maize streak virus evolution. BMC Evolutionary Biology 2011 11:350.

\section{Submit your next manuscript to BioMed Central and take full advantage of:}

- Convenient online submission

- Thorough peer review

- No space constraints or color figure charges

- Immediate publication on acceptance

- Inclusion in PubMed, CAS, Scopus and Google Scholar

- Research which is freely available for redistribution

Submit your manuscript at www.biomedcentral.com/submit
Biomed Central 